\title{
Performance Analysis of Eigenvalue Based Spectrum Sensing under Frequency Selective Channels
}

\author{
Sener Dikmese and Markku Renfors \\ Department of Communications Engineering \\ Tampere University of Technology \\ Tampere, Finland \\ sener.dikmese@tut.fi and markku.renfors@tut.fi
}

\begin{abstract}
Detecting primary signals with very low Signal-toNoise Ratio (SNR) is a very important problem in cognitive radio (CR) systems. Small parameter uncertainties are unavoidable in any practical system, and especially the noise variance uncertainty has great effect on the performance of the most basic spectrum sensing method, energy detection. This has motivated the need for advanced spectrum sensing algorithms, like eigenvalue based spectrum sensing, which can be used to overcome the effects of parameter uncertainty in very low SNR cases. In this paper we study the effects of channel frequency selectivity, in combination with noise uncertainty, in case of energy detector and eigenvalue based spectrum sensing. It is demonstrated that channel frequency selectivity significantly enhances the performance of eigenvalue based spectrum sensing techniques.
\end{abstract}

Keywords-component; Energy detector based spectrum sensing, eigenvalue based spectrum sensing, $A W G N$, frequency selective channel and noise uncertainty

\section{INTRODUCTION}

$\mathrm{I}_{\mathrm{i}}^{\mathrm{n}}$ ncreasing traffic rates, limited system capacity and inefficient spectrum utilization are very important challenges in the future development of wireless communications [1]. In solving these challenges, cognitive radio (CR) and advanced signal processing techniques have recently been studied extensively [2, 3, 4, 5, 6]. Spectrum sensing is a fundamental component of CR systems. Hence, different spectrum sensing algorithms have been developed with different characteristics regarding detection sensitivity and tolerance against various imperfections which are unavoidable in practical sensing devices.

There are many problems which affect the performance of spectrum sensing in practice. The first problem is that reliable sensing has to be achieved with very low signal-to-noise ratio (SNR). Secondly, the multipath fading and shadowing cause power fluctuation of the received signal [7]. Variation and unpredictability of the precise noise level at the sensing device is another critical issue, which is called "noise uncertainty". In many studies, the noise variance is assumed to be exactly known according to previous measurements [8], but in practice it is often very difficult to estimate the noise level accurately. Especially, the performance of the traditional energy detector based spectrum sensing methods significantly decreases under noise uncertainty [7].
Alternative spectrum sensing methods have been investigated to overcome these challenges. Eigenvalue and covariance based spectrum sensing techniques are very interesting alternative solutions for the noise uncertainty case, in spite of relatively high computational complexity. While the noise variance knowledge is not required for eigenvalue and covariance based spectrum sensing techniques, small changes or uncertainty on noise variance have no effect on the spectrum sensing performance $[9,10]$.

So far, most of the spectrum sensing studies have focused on the AWGN channel model. While most of CR systems work under frequency selective channel, investigation of frequency selective channel effects on spectrum sensing algorithms is a very important topic.

The goal of this paper is to investigate the effects of frequency selective channel, considering also the noise uncertainty effects, using traditional energy detector and eigenvalue based spectrum sensing. We consider a simplified signal scenario, where only Gaussian signal model is used under Indoor, SUI-1 and ITU-R Vehicular A multipath delay profiles [11]. The applications of cooperative sensing approaches, which are very essential for reliable overall spectrum sensing schemes, are left as topics for future studies.

The rest of the paper is organized as follows. In Section 2, signal models are given for different frequency selective channels and analysis of energy detector and eigenvalue based spectrum sensing are summarized. Section 3 gives simulation results for the channel models considered, and finally, some concluding remarks are given about the performance of these methods and about the effects of channel frequency selectivity on the performance of eigenvalue based spectrum sensing

\section{Spectrum Sensing Techniques}

\section{A. Energy detector based spectrum sensing with noise uncertainty}

The analysis of energy detector based spectrum sensing, considering also the effects of noise uncertainty, is given in this section based on [7]. The two hypotheses regarding the absence or presence of a primary transmission in the received signal, can be expressed as 


$$
\begin{aligned}
& H 0: y(n)=w(n) \sim N\left(0, \sigma_{w}^{2}\right) \\
& H 1: y(n)=\overbrace{s(n) \otimes c(n)}^{x(n)}+w(n) \sim N\left(0, \sigma_{x}^{2}+\sigma_{w}^{2}\right)
\end{aligned}
$$

When the AWGN only is present, the white noise is modeled as a zero-mean Gaussian random variable with variance $\sigma_{w}^{2}$, i.e., $w=N\left(0, \sigma_{w}^{2}\right)$. Signal can also be modeled as a zero-mean Gaussian variable $x=N\left(0, \sigma_{x}^{2}\right)$ where, $\sigma_{x}^{2}$ is the variance (power) of the received primary user signal, including the effects of the channel fading and frequency selectivity.

For this case, the decision statistics can be obtained as [3]

$$
T_{y}=\frac{1}{N} \sum_{n=0}^{N-1}|y[n]|^{2}
$$

As $y$ has complex Gaussian distribution, the probability distribution functions (PDF) of the outputs of test statistic $T_{y}$ can be approximated as Gaussian distributions under $H_{0}$ and $H_{1}$ [7]. Hence, the probability distribution of test statistic $T_{y}$ can be modeled as

$$
\begin{aligned}
& \left.T_{y}\right|_{H 0} \sim N\left(\sigma_{w}^{2}, \frac{1}{N} \sigma_{w}^{4}\right) \\
& \left.T_{y}\right|_{H 1} \sim N\left(\sigma_{x}^{2}+\sigma_{w}^{2}, \frac{1}{N}\left(\sigma_{x}^{2}+\sigma_{w}^{2}\right)^{2}\right)
\end{aligned}
$$

There are two vital probabilities, the probability of detection $P_{D}$ and probability of false alarm $P_{F A}$. When there is a signal in the sensing spectrum interested, $P_{D}$ is considered and it can be defined as

$$
P_{D}=P\left(T_{y}>\gamma \mid H_{1}\right)
$$

where $\lambda$ is the threshold value for detection. The false alarm probability $P_{F A}$ gives the probability for the event that the primary signal is absent, but the decision device decides incorrectly that there is a signal. It can be formulated as [3]

$$
P_{F A}=P\left(T_{y}>\gamma \mid H_{0}\right)
$$

The threshold value can be obtained according to the target false alarm probability and noise variance assumed. The threshold value and the actual false alarm and detection probabilities can be expressed as follows

$$
\begin{gathered}
\gamma=Q^{-1}\left(P_{F A}\right) \sqrt{\frac{1}{N} \sigma_{w}^{4}}+\sigma_{w}^{2} \\
P_{F A}=P\left(T_{y}>\gamma \mid H_{0}\right)=Q\left(\left(\gamma-\sigma_{w}^{2}\right) / \sqrt{\frac{1}{N} \sigma_{w}^{4}}\right) \\
P_{D}=P\left(T_{y}>\gamma \mid H_{1}\right)=Q\left(\frac{\gamma-\left(\sigma_{x}^{2}+\sigma_{w}^{2}\right)}{\sqrt{\frac{1}{N}\left(\sigma_{x}^{2}+\sigma_{w}^{2}\right)^{2}}}\right)
\end{gathered}
$$

The optimum threshold value $\lambda$ is calculated according to the noise and primary signal variances information. It is very difficult to actually know the signal variance due to the channel environment characteristics. Hence, threshold value always is calculated from the noise variance which is assumed to be known according to the previous measurements or special noise calibration techniques possibly applied in the receiver. In practice, the estimation of exact noise variance is not possible. The detection and false alarm probabilities as functions of the SNR depend critically on the accuracy of noise variance estimate. In practice, the noise variance can be expected to be in the range $\sigma_{w}^{2} \in\left[(1 / \rho) \sigma_{w}^{2}, \rho \sigma_{w}^{2}\right]$ where $\rho>1$ is a parameter that quantizes the size of the uncertainty. The noise uncertainty is usually expressed in $\mathrm{dB}$ units as $x=10 \log _{10} \rho$. In the presence of noise uncertainty, the expressions for $P_{F A}$ and $P_{D}$ are modified as follows [7]

$$
\begin{gathered}
P_{F A}=\max _{\sigma^{2} \in\left[\frac{1}{\rho} \sigma_{w}^{2}, \rho \sigma_{w}^{2}\right]} Q\left(\left(\gamma-\sigma^{2}\right) / \sqrt{\left.\frac{1}{N}\left(\sigma^{2}\right)^{2}\right)}\right. \\
=Q\left(\left(\gamma-\rho \sigma_{w}^{2}\right) / \sqrt{\left.\frac{1}{N}\left(\rho \sigma_{w}^{2}\right)^{2}\right)}\right. \\
P_{D}=\min _{\sigma^{2} \in\left[\frac{1}{\rho} \sigma_{w}^{2}, \rho \sigma_{w}^{2}\right]} Q\left(\left(\gamma-\left(\sigma_{x}^{2}+\sigma^{2}\right)\right) / \sqrt{\left.\frac{1}{N}\left(\sigma_{x}^{2}+\sigma^{2}\right)^{2}\right)}\right. \\
=Q\left(\frac{\gamma-\left(\sigma_{x}^{2}+(1 / \rho) \sigma_{w}^{2}\right)}{\sqrt{\frac{1}{N}\left(\sigma_{x}^{2}+(1 / \rho) \sigma_{w}^{2}\right)^{2}}}\right)
\end{gathered}
$$

The noise uncertainty introduces the so-called SNR wall: with a given uncertainty $\rho$ there is a minimum SNR value under which a primary signal cannot be reliably detected, no matter how long time record is used.

\section{B. Eigenvalue based spectrum sensing}

Eigenvalue based spectrum sensing algorithms can be applied for different kind of signals without noise variance knowledge. Hence, these algorithms are very robust, overcoming the noise uncertainty problem, and can even perform better than energy detection when the signals to be detected are highly correlated. In the following we review the eigenvalue based algorithms based on $[9,10]$.

The signal model of eq. (1) is valid also in this case. We considering $L$ consecutive symbol intervals with $M$ samples within each interval. Within each symbol interval, the signal is highly correlated. $M$ is called the oversampling factor. Now the sequences of received signal, primary signal, and noise are defined as

$$
\begin{aligned}
& \hat{\mathbf{y}}=\left[\begin{array}{lll}
y(n) & y(n-1) & y(n-2) \ldots y(n-M L+1)
\end{array}\right]^{T}, \\
& \hat{\mathbf{s}}=\left[\begin{array}{lll}
s(n) & s(n-1) & s(n-2) \ldots s(n-M L+1)
\end{array}\right]^{T}, \\
& \hat{\mathbf{w}}=\left[\begin{array}{lll}
w(n) & w(n-1) & w(n-2) \ldots w(n-M L+1)
\end{array}\right]^{T}
\end{aligned}
$$

The statistical covariance matrices of the signal and noise are defined as

$$
\begin{aligned}
& \mathbf{R}_{y y}=E\left(\hat{\mathbf{y}} \hat{\mathbf{y}}^{\dagger}\right) \\
& \mathbf{R}_{s s}=E\left(\hat{\mathbf{s}} \hat{\mathbf{s}}^{\dagger}\right) \\
& \mathbf{R}_{w w}=E\left(\hat{\mathbf{w}} \hat{\mathbf{w}}^{\dagger}\right) \\
& \mathbf{R}_{y y}=\mathbf{H} \mathbf{R}_{s s} \mathbf{H}^{\dagger}+\mathbf{R}_{w w}
\end{aligned}
$$

Eigenvalues of $\mathbf{R}_{y y}$ and $\mathbf{H} \mathbf{R}_{s s} \mathbf{H}^{\dagger}$ are defined as $\lambda_{1} \geq \lambda_{2} \geq \ldots \geq \lambda_{M L}$ and $\rho_{1} \geq \rho_{2} \geq \ldots \geq \rho_{M L}$, respectively. 
1) Algorithm 1: Max-Min eigenvalue based sensing (MME)

Compute the maximum and minimum eigenvalues $\left(\lambda_{\max }, \lambda_{\min }\right)$ of the covariance matrix $\mathbf{R}_{w}(N)$. The covariance matrix is obtained by averaging $N$ sample covariance matrices (here $n$ indicates the first sample used in the calculation of each covariance estimate)

$$
\mathbf{R}_{y y}(N)=\frac{1}{N} \sum_{n=M L-1}^{L-2+N} \hat{\mathbf{y}}(n) \hat{\mathbf{y}}(n)^{\dagger}
$$

The ratio of $\lambda_{\max }, \lambda_{\min }$ is compared with the threshold $\gamma_{1}$ which is calculated according to the distribution of covariance matrix of noise, when the signal is absent

$$
\mathbf{R}_{w w}(N)=\frac{1}{N} \sum_{n=M L-1}^{L-2+N} \hat{\mathbf{w}}(n) \hat{\mathbf{w}}(n)^{\dagger}
$$

$\mathbf{R}_{w w}(N)$ is nearly a Wishart random matrix [12]. The distribution of the eigenvalues has been investigated to define the threshold value. In $[13,14]$ the Tracy-Widom distributions were studied and $F_{1}$, the cumulative distribution function (CDF) of the Tracy-Widom distribution of order 1 was derived to get closed form expression

$$
F_{1}=\exp \left(-\frac{1}{2} \int_{t}^{\infty}\left(q(u)+(u-t) q^{2}(u)\right) d u\right)
$$

where $q(u)$ is the solution of the nonlinear Painleve II differential equation

$$
q^{\prime \prime}(u)=u q(u)+2 q^{3}(u)
$$

Table 1 gives the values of $F_{1}$ at some points. Also $F_{1}^{-1}$ can calculated using same table

TABLE I

NUMERICAL TABLE FOR THE TRACY-WIDOM DIST. OF ORDER 1

\begin{tabular}{|c|c|c|c|c|c|c|c|c|c|}
\hline $\mathrm{t}$ & -3.90 & -3.18 & -2.78 & -1.91 & -1.27 & -0.59 & 0.45 & 0.98 & 2.02 \\
\hline $\mathrm{F}_{1}(\mathrm{t})$ & 0.01 & 0.05 & 0.10 & 0.30 & 0.50 & 0.70 & 0.90 & 0.95 & 0.99 \\
\hline
\end{tabular}

Utilizing the table or numerical method for calculating the values for $F_{1}^{-1}$, together with values of $N$ and $L$, the threshold $\gamma_{1}$ can be formulated as

$$
\gamma_{1}=\frac{(\sqrt{N}+\sqrt{M L})^{2}}{(\sqrt{N}-\sqrt{M L})^{2}} \cdot\left(1+\frac{(\sqrt{N}+\sqrt{M L})^{-2 / 3}}{(N M L)^{1 / 6}} F^{-1}\left(1-P_{F A}\right)\right)
$$

When $\left(\lambda_{\max } / \lambda_{\min }\right)>\gamma_{1}$, the primary signal is deemed to be present, otherwise it is assumed that there is no signal in the band of interest.

It is very intractable mathematically to get theoretical detection probabilities for the $\mathrm{max} / \mathrm{min}$ eigenvalue based spectrum sensing algorithm. Hence, approximated value has been obtained using an empirical mode [9]. This approximation is given for Algorithm1 as follows

$$
\mathbf{R}_{y y}=\mathbf{H} \mathbf{R}_{s s} \mathbf{H}^{\dagger}+\mathbf{R}_{w w}=\mathbf{H} \mathbf{R}_{s s} \mathbf{H}^{\dagger}+\sigma_{w}^{2} \mathbf{I}
$$

$$
\begin{aligned}
& \lambda_{\text {max }}\left(\mathbf{R}_{x x}(N)\right) \approx \rho_{1}+\lambda_{\text {max }}\left(\mathbf{R}_{w w}(N)\right) \\
& \lambda_{\text {min }}\left(\mathbf{R}_{x x}(N)\right) \approx \rho_{M L}+\sigma_{w}^{2} \\
& P_{d}=P\left(\lambda_{\max }\left(\mathbf{R}_{x x}(N)\right)>\gamma_{1} \lambda_{\min }\left(\mathbf{R}_{x x}(N)\right)\right) \\
& \quad \approx P\left(\lambda_{\max }\left(\mathbf{R}_{w w}(N)\right)>\gamma_{1}\left(\rho_{M L}+\sigma_{w}^{2}\right)-\rho_{1}\right. \\
& \quad=1-F_{1}\left(\frac{\gamma_{1} N+N\left(\gamma_{1} \rho_{M L}-\rho_{1}\right) / \sigma_{w}^{2}-\mu}{v}\right)
\end{aligned}
$$

In equation (20), according to the random matrix theorem [12], $\mu$ and $v$ are calculated as $(\sqrt{N-1}+\sqrt{M L})^{2}$ and $(\sqrt{N-1}+\sqrt{M L})((1 / \sqrt{N-1})+(1 / \sqrt{M L}))^{1 / 3}$.

2) Algorithm 2: Energy with min eigenvalue based sensing $(E M E)$

Compute the minimum eigenvalue $\lambda_{\min }$ of the covariance matrix $\mathbf{R}_{y y}(N)$ in the same way with Algorithm 1. Then, compute average power of the received signal as

$$
T(N)=\frac{1}{M N} \sum_{n=0}^{N M-1}|y(n)|^{2}
$$

Threshold value $\gamma_{2}$ is calculated with the inverse q-function $Q^{-1}$ as follows

$$
\gamma_{2}=\left(\sqrt{\frac{1}{M N}} Q^{-1}\left(P_{F A}\right)+1\right) \frac{N}{(\sqrt{N}-\sqrt{M L})^{2}}
$$

When $\left(T(N) / \lambda_{\min }\right)>\gamma_{2}$, the signal is assumed to be present, otherwise it is expected that there is no signal in the band of interest.

While the thresholds can be pre-computed based only on $N$ , $L$ and $P_{F A}$, there is no need to estimate noise variance according to the previous measurements. Hence, it can be seen that these two algorithms are very robust to noise uncertainty.

Similar theoretical difficulties are encountered when calculating the probability of detection for Algorithm 2 . However, the following numerical expression has been established [9]

$$
\begin{aligned}
& P_{d}=P\left(T(N)>\gamma_{2} \lambda_{\text {min }}\left(\mathbf{R}_{x x}(N)\right)\right) \\
& \approx P\left(\frac{\operatorname{Tr}\left(\mathbf{R}_{w w}(N)\right)}{M L}>\gamma_{2}\left(\rho_{M L}+\frac{\sigma_{w}^{2}}{\sqrt{N}}(\sqrt{N}-\sqrt{M L})\right)-\left(\frac{\operatorname{Tr}\left(\mathbf{H} \mathbf{R}_{s s} \mathbf{H}^{\dagger}\right)}{M L}\right)\right) \\
& \left.=q \frac{\gamma_{2}\left(\rho_{M}+\frac{\sigma_{w}^{2}}{\sqrt{N}}(\sqrt{N}-\sqrt{M \mathbb{L}})\right)-\left(\frac{\operatorname{Tr}(\mathbf{H R} \mathbf{H} \mathbf{H})}{M \mathbb{L}}\right)-\sigma_{w}^{2}}{\sqrt{\frac{1}{M N}} \sigma_{w}^{2}}\right)
\end{aligned}
$$

Due to the approximation, there are some differences between theoretical and simulation results of the two algorithms.

The effects of channel frequency selectivity appear into the detection probability expressions (20) and (23) through the eigenvalues. The eigenvalue spread is a metric for the correlatedness of the sample sequence used in detection. Naturally, flat signal or noise spectra correspond to uncorrelated sample sequences, in which case the covariance matrix approaches a scaled unit matrix and the eigenvalues are 
identical. Correlations are introduced to the possible primary signal spectrum through the characteristics of the transmitted waveform, e.g., by pulse shaping or channelization filtering. Additionally, frequency selective channel introduces correlations (i.e., a non-flat power spectrum) to the received sample sequence. With non-oversampled signal model, the waveform generation related correlations disappear if the signal spectrum is flat within the used signal band. Still the channel frequency selectivity based correlations may be sufficient for detecting the primary signal. This idea is tested in the following through numerical examples.

\section{SIMULATION RESULTS}

Simple Gaussian signal models which includes both nonoversampled and $2 \mathrm{x}$-oversampled signal are shown as seen figure 1. In this figure, the signals are shown for the ITU-R Vehicular A channel case [11]. In our signal model, the bandwidth is chosen as $20 \mathrm{MHz}$. The Vehicular A channel model has 6 taps the maximum delay spreads is about $2.5 \mu \mathrm{s}$.

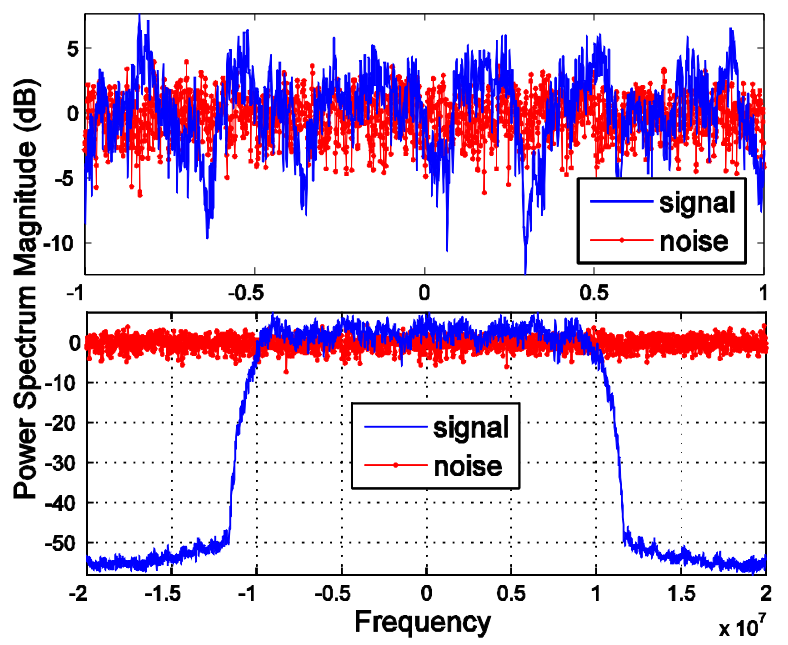

Figure 1. Examples non-oversampled and 2x-oversampled spectral models under Vehicular A channel and AWGN noise.

For realistic Indoor channel model, we use the 16-tap model with $80 \mathrm{~ns}$ rms delay spread such as from [15]. The third channel model used in this study is the SUI-1 model [11]. This model has 3 Ricean fading taps and $0.9 \mu$ s delay spread, and it is clearly less frequency selective than the other two. These different cases are presented in the following subsections. Theoretical results with channel effects are obtained using the model of eqs. (9) and (10) for energy detection with noise uncertainty and eqs. (20) and (23) for eigenvalue based spectrum sensing. The simulation results, as well as theoretical results are averaged over 1000 channel instances.

\section{A. Indoor channel case for both non-oversampled and oversampled Gaussian signal model}

Figure 2 shows detection probabilities of traditional energy detector and eigenvalue based spectrum sensing with Indoor channel [15] in the non-oversampled case. The $1 \mathrm{~dB}$ noise uncertainty case is considered as the worst-case scenario in terms of channel noise variance estimation. The time record length is 10000 complex samples. With this number of samples and $1 \mathrm{~dB}$ noise uncertainty, eigenvalue based spectrum sensing has still better performance compared to the energy detector based spectrum sensing with frequency selective channel. Similar results are shown for $2 \mathrm{x}$-oversampled signal using the same channel instances as in figure 3 .

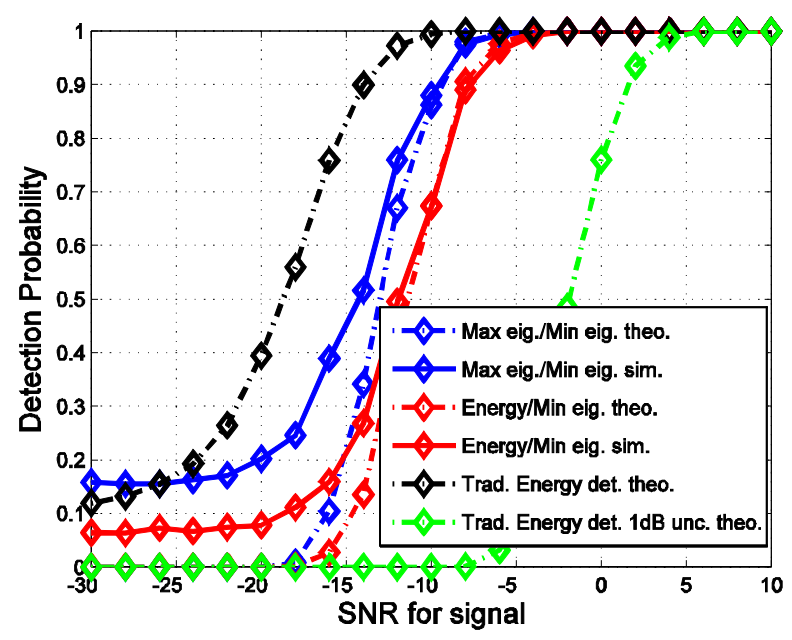

Figure 2. Detection probability of energy detector and eigenvalue based spectrum sensing under $1 \mathrm{~dB}$ uncertainty using Indoor channel with nonoversampled signal.

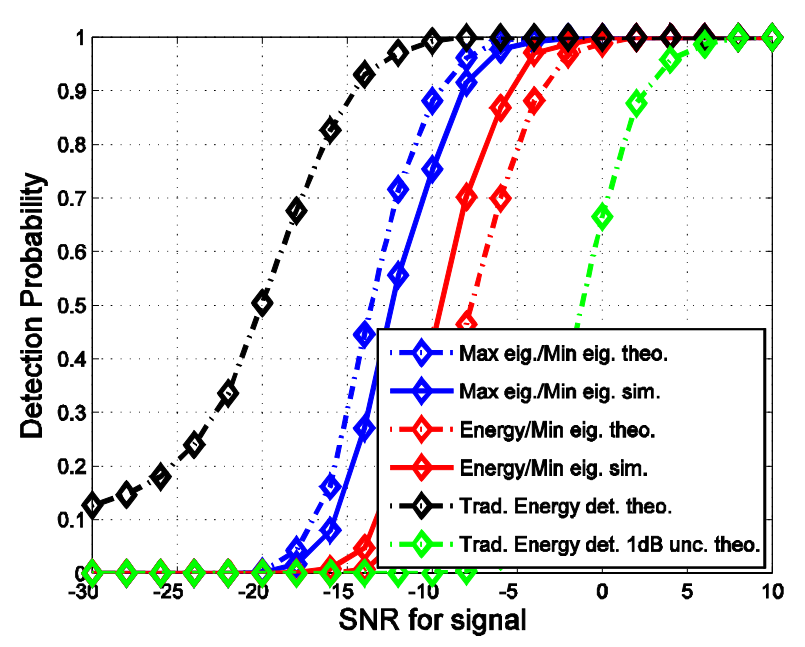

Figure 3. Detection probability of tenergy detector and eigenvalue based spectrum sensing under $1 \mathrm{~dB}$ uncertainty using Indoor channel with $2 \mathrm{x}$ oversampled signal.

The actual false alarm probabilities of eigenvalue based spectrum sensing for both non-oversampled and oversampled signals are given in figure 4 . We notice that the false alarm probability is independent of SNR, as expected $[9,10]$. While the simulated false alarm probability in the non-oversampled signal case becomes quite significant, the actual false alarm probability under oversampled signal model becomes very 
small. Very similar performance is obtained also for SUI-1 and Vehicular A channel cases, as can be seen from the 'floors' of the detection performance curves. Thus the actual false alarm probability is not related to channel effects in eigenvalue based spectrum sensing. The same detection threshold is used in non-oversampled and oversampled cases. Based on these results, there is the possibility to reduce the detection threshold in the oversampled case to improve the detection performance, while maintaining a realistic false alarm probability.

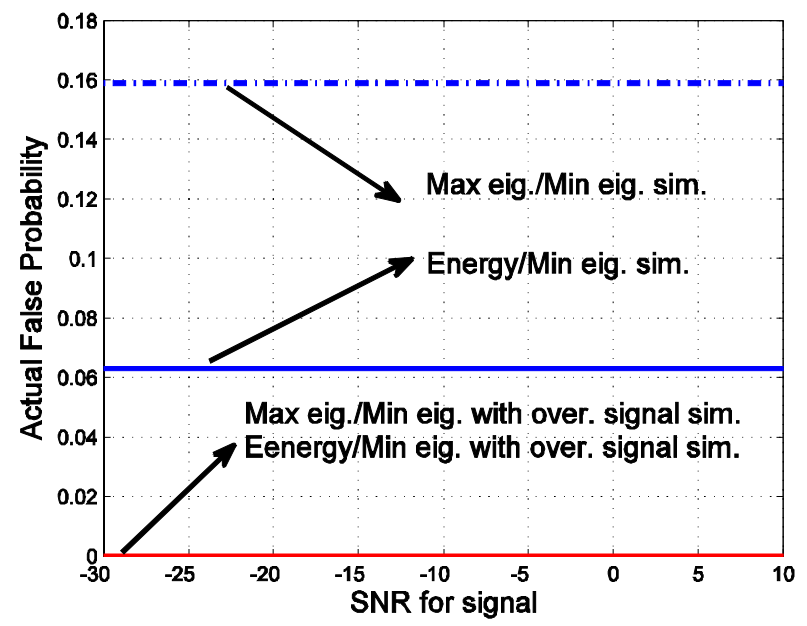

Figure 4. Actual false alarm probability of eigenvalue based spectrum sensing techniques.

B. Vehicular A channel case for both non-oversampled and oversampled Gaussian signal model

Vehicular A channel model [11] is applied for both nonoversampled and oversampled signal models in figure 5 and figure 6 , respectively. The same signal parameters are used as in the Indoor channel case. Significant performance differences can be seen due to the difference of channel models.

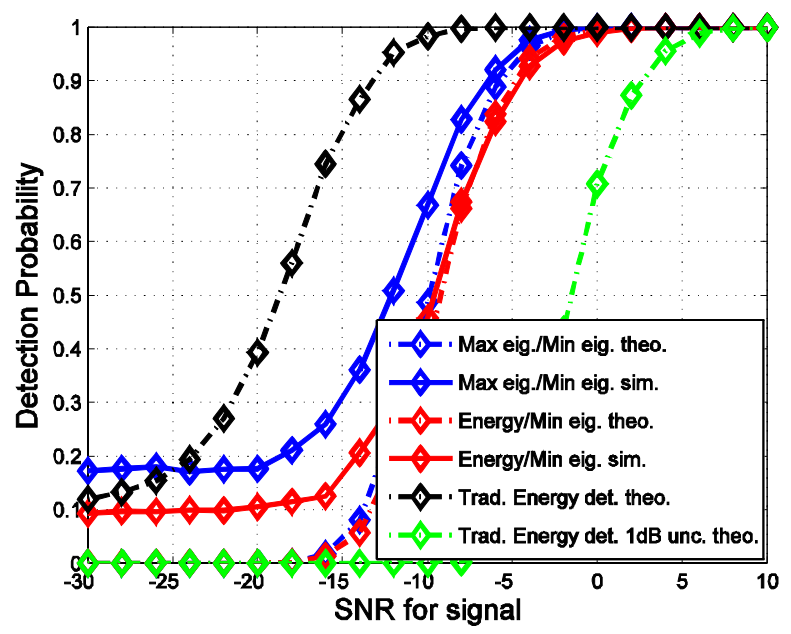

Figure 5. Detection probability of energy detector and eigenvalue based spectrum sensing under $1 \mathrm{~dB}$ uncertainty using Vehicular A channel with non-oversampled signal.

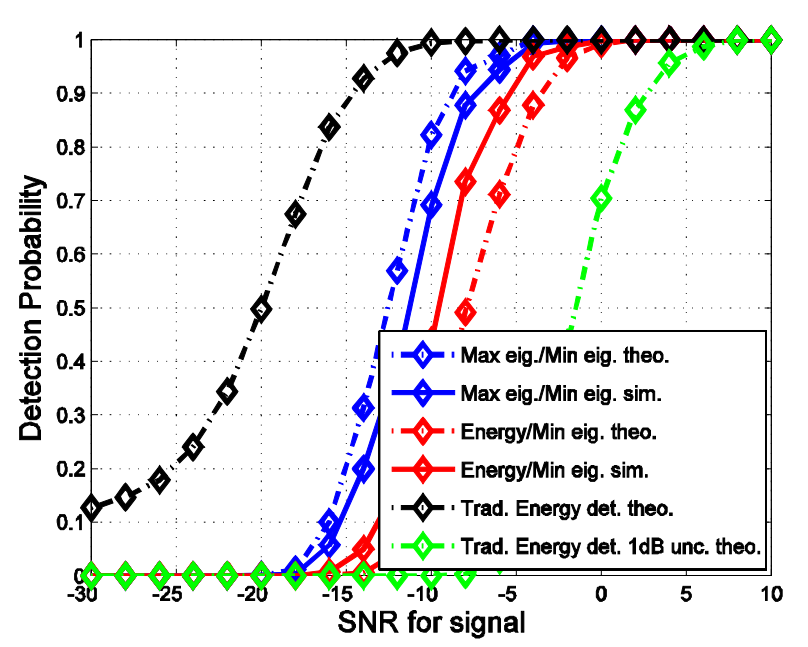

Figure 6. Detection probability of energy detector and eigenvalue based spectrum sensing under $1 \mathrm{~dB}$ uncertainty using Vehicular A channel with oversampled signal.

C. SUI-1 channel case for both non-oversampled and oversampled Gaussian signal model

In figures 7 and 8, the channel model is chosen as SUI-1 [11] for both non-oversampled and oversampled signal models, respectively. Comparing the results of non-oversampled and oversampled signal cases, significant difference of detection probability can be seen with eigenvalue based spectrum sensing. In the oversampled signal case, the correlation between consecutive samples is increased. In case of traditional energy detector, the detection performance is somewhat reduced with oversampling due to increased noise bandwidth.

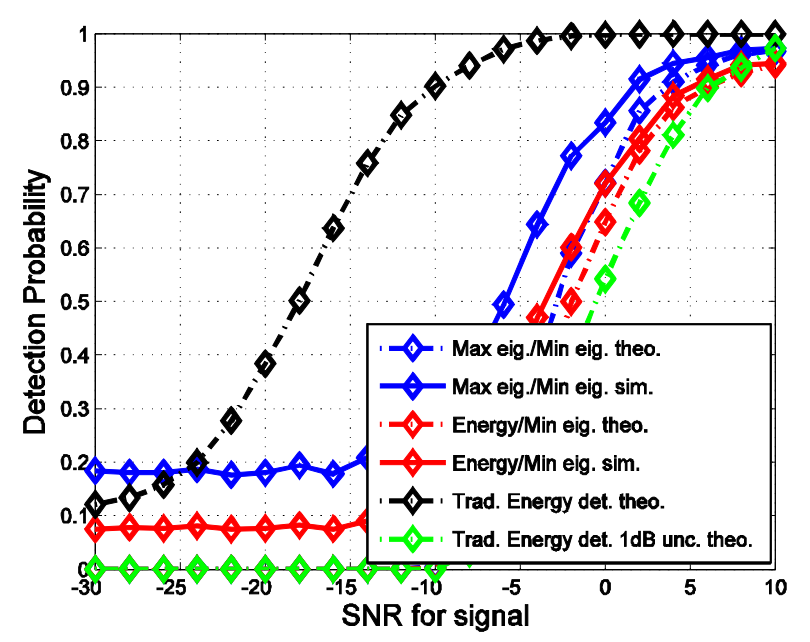

Figure 7. Detection probability of energy detector and eigenvalue based spectrum sensing under $1 \mathrm{~dB}$ uncertainty using SUI-1 channel with nonoversampled signal. 


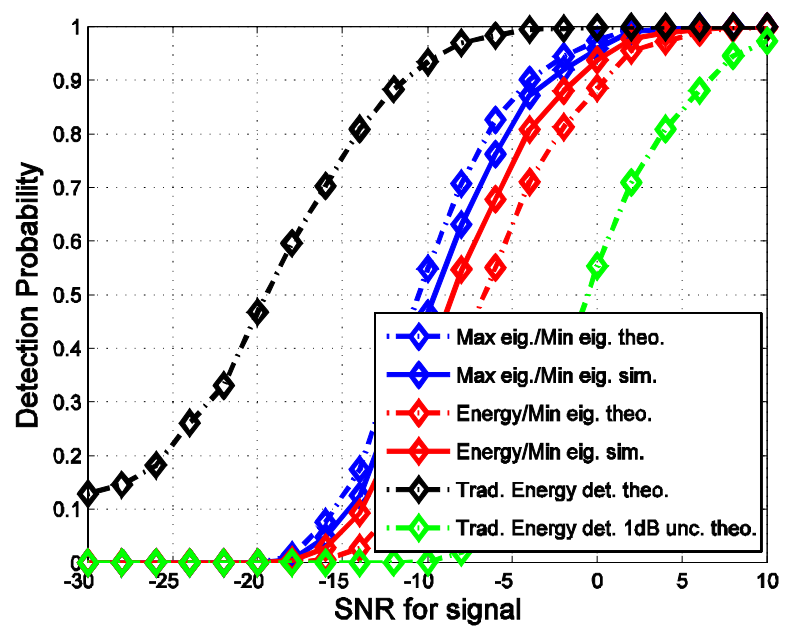

Figure 8. Detection probability of energy detector and eigenvalue based spectrum sensing under $1 \mathrm{~dB}$ uncertainty using SUI-1 channel with oversampled signal.

\section{CONCLUSIONS}

We have analyzed the performance of the traditional energy detector and eigenvalue based spectrum sensing techniques under different frequency selective channels, the Indoor, ITU-R Vehicular A and SUI-1 channel models in particular. Actually, the frequency selective channel can help to increase detection probability when using eigenvalue or covariance based spectrum sensing algorithms.

It was seen that $\mathrm{max} / \mathrm{min}$ eigenvalue approach gives consistently better detection performance that energy/min eigenvalue approach. Especially, in simulation based results with oversampling the difference is significant.

We have seen that eigenvalue based spectrum sensing clearly exceeds the performance of energy detector with $1 \mathrm{~dB}$ noise uncertainty with Indoor and Vehicular-A channel models, whereas with SUI-1, the difference is rather small. Using oversampled signal model in detection clearly reduces the false alarm probability with eigenvalue based sensing. With SUI-1, also the detection performance is significantly improved when oversampled signal is used in eigenvalue based detection, whereas with Indoor and Vehicular-A channel models, the detection performance is slightly degraded in the oversampled case.

The above observations are explained by the fact that SUI-1 is much less frequency selective than the other channel models used. Thus in this case the channel creates less correlations to the received signal, and the correlations due to the spectral shaping of the transmitted signal are much more important in the eigenvalue based detection than in the more frequency selective cases.

One related general aspect regarding spectrum sensing is the following: When the sensing station has a line-of sight (LOS) connection, the channel can be expected to be mildly frequency selective, but also the power level is high due to lower path loss. When the sensing station does not have a LOS connection, the signal level is lower, but also the channel can be expected to be highly frequency selective. Thus, in case of shadowing, the PU signal can be detected using the eigenvalue based approach without essential limitations due to noise uncertainty. In case of LOS channel, simple energy detection based approach might be sufficient.

While Gaussian signal model is used in this study, similar techniques and similar conclusions can be also applied for spectrum sensing with other primary systems. In the future work, to complete the picture, we will consider the effects of the spectrum sensing using real-life signal models such as WLAN and Bluetooth. It would be interesting also to quantify analytically the correlations introduced by the waveform and channel in different scenarios. Another topic is to apply lowcomplexity covariance matrix based methods, instead of the relatively complicated eigenvalue based approaches.

\section{ACKNOWLEDGMENT}

This work was partially supported by Tekniikan Edistämissäätiön (TES), GETA Graduate School and Tekes (the Finnish Funding Agency for Technology and Innovation) under the project ENCOR in the Trial Program.

\section{REFERENCES}

[1] I. Mitola, J. and J. Maguire, G. Q., "Cognitive radio: making software radios more personal," IEEE Personal Commun. Mag., vol. 6, no. 4, pp. 13-18, Aug. 1999.

[2] Y. Zeng, Y.C. Liang, A. T. Hoang, and R. Zhang, "A Review on Spectrum Sensing for Cognitive Radio: Challenges and Solutions," EURASIP Jor. on Ad. in Sig. Proc, vol. 2010, pp. 1-15, Jan. 2010.

[3] T. Yucek and H. Arslan, "A survey of spectrum sensing algorithms for cognitive radio applications," IEEE Communications Surveys \& Tutorials, vol. 11, no. 1, pp. 116-130, March 2009.

[4] S. Dikmese, M. Renfors and H. Dincer, "FFT and Filter Bank Based Spectrum Sensing for WLAN Signals," in Proc. ECCTD2011 conf., Linkoping, Sweden, August 2011.

[5] S. Dikmese and M. Renfors, "Optimized FFT and Filter Bank Based Spectrum Sensing for Bluetooth Signal" in Proc. WCNC2012, Paris, France, April 2012.

[6] S. Dikmese and S. Srinivasan and M. Renfors, "FFT and Filter Bank Based Spectrum Sensing and Spectrum Utilization for Cognitive Radios" in Proc. Int. Symposium on Comm., Cont. and Sig. Proc. (ISCCSP 2012), Rome, Italy.

[7] R.Tandra and A. Sahai, "SNR walls for signal detection," IEEE J. Selected Topics Signal Processing, vol. 2, pp. 4-17, February 2008.

[8] S. Geirhofer, L. Tong, and B. Sadler, "A measurement-based model for dynamic spectrum access in WLAN channels," in Proc. IEEE MilitaryCommun. Conf., Washington, D.C., USA, Oct. 2006.

[9] Y. H. Zeng and Y.-C. Liang, "Eigenvalue-based spectrum sensing algorithms for cognitive radio," IEEE Transactions on Communications, vol. 57, no. 6, pp. 1784-1793, 2009.

[10] Y. H. Zeng and Y.-C. Liang, "Spectrum-sensing algorithms for cognitive radio based on statistical covariances," IEEE Transactions on Vehicular Technology, vol. 58, no. 4, pp. 1804-1815, 2009.

[11] R. Jain, "Channel Models: A Tutorial," WiMAX Forum AATG, February 2007.

[12] A. M. Tulino and S. Verdu, "Random Matrix Theory and Wireless Communications" Now Publishers Inc., 2004.

[13] C. A. Tracy and H. Widom, "On orthogonal and symplectic matrix ensembles," Commun. Math. Phys., vol.177, pp. 727-754, 1996.

[14] C. A. Tracy and H. Widom, "The distribution of the largest eigenvalue in the gaussian ensembles," in Calogero-Moser-Sutherland Models, J. van Diejen and L. Vinet, eds., pp. 461-472. Newyork: Springer, 2000.

[15] T.Yucek, "Channel, Spectrum, and Waveform Awareness in OFDMBased Cognitive Radio Systems” Ph.D. thesis, Tampa, 2007 